\title{
Rationalités et gestion de contraintes dans l'innovation : l'exemple de la Plateforme du bâtiment
}

\section{Rationality and constraint management in the innovation process: the case of Saint Gobain's Plateforme du bâtiment}

\author{
Joëlle Forest ${ }^{1}$, Ludovic Vievard ${ }^{2}$ \\ ${ }^{1}$ EA 4148 - S2HEP, Université de Lyon, INSA Lyon, France, joelle.forest@insa-lyon.fr \\ ${ }^{2}$ Chargé de recherche, chaire de recherche Ingénieurs ingénieux, INSA Lyon, Membre fondateur de l'agence de sciences \\ humaines appliquées FRV100, ludovic.vievard@gmail.com
}

RÉSUMÉ. L'injonction permanente à innover pose avec acuité la question de la compréhension du mode d'existence des innovations. Comment émerge en effet les innovations qui façonnent notre monde et nos comportements ? Afin d'apporter des éléments de réponse, cet article revient sur l'histoire d'une innovation marketing à savoir la genèse de la plateforme du bâtiment de Saint Gobain. S'il apparait clairement que la genèse de cette innovation n'émerge pas ex nihilo, l'histoire de la plateforme du bâtiment permet de constater que la forme finale prise par cette innovation n'est pas étrangère à la façon dont les concepteurs ont pris en charge les contraintes inhérentes à tout processus de conception. L'histoire de la Plateforme du bâtiment met par ailleurs en exergue le fait que l'innovation résulte de la mise en œuvre de deux formes de pensée : la rationalité créative et analytique. Chemin faisant, cet article nous conduit à nous émanciper d'une conception romantique de l'innovation héritée d'une mise en récit magnifiée de la genèse des grandes innovations. ABSTRACT. The permanent injunction to innovate raises the question of the mode of existence of innovations. How do the innovations that shape our world and our behaviors emerge? In order to provide some answers, this article reviews the history of a marketing innovation: the plateforme du bâtiment of Saint Gobain. Although it is clear that the genesis of this innovation does not emerge ex nihilo, the history of the plateforme du bâtiment shows that the final form taken by this innovation is closely linked to the way the designers took into account the constraints inherent in any design process. It also highlights the fact that innovation results from the deployment of two forms of thought: creative and analytical rationality. In this way, this article encourages us to emancipate ourselves from a romantic conception of innovation inherited from the magnified storytelling of the genesis of great innovations.

MOTS-CLÉS. contrainte, rationalité créative, rationalité analytique, innovation, Saint Gobain, Plateforme du bâtiment. KEYWORDS. constraint, creative rationality, analytical rationality, innovation, Saint Gobain, Plateforme du bâtiment.

\section{Introduction}

Au milieu des années 1990, le groupe Poliet, qui appartient à Paribas, est le leader français de la distribution de matériaux, notamment via ses marques Point P et Lapeyre. Pour préparer l'avenir du groupe, Jean-Gérard Claudon, arrivé en 1992 à la présidence du Groupe, forme un groupe de travail «innovation» afin d'explorer l'évolution des modèles de diffusion des matériaux de construction. L'objectif est de déterminer une $4^{\mathrm{e}}$ voie, venant compléter le négoce généraliste (dont Point $\mathrm{P}$ est le leader), le négoce spécialisé (type Lapeyre), et les Grandes Surfaces de Bricolage (GSB). L'enjeu est de se préparer à la concurrence qui monte surtout du côté des GSB, Castorama et Leroy Merlin en tête «qui captent la partie des particuliers qui viennent dans le négoce » [CAR 18]. Le groupe de réflexion ainsi constitué compte des cadres dirigeants de Lapeyre, KparK, GME et Point P. On y trouve notamment Gérard Aspar, alors directeur général de Lapeyre. C'est lui qui, fin 1995, est chargé par Jean-Gérard Claudon de développer le concept, lequel sera validé dans sa forme définitive en septembre 1996 par Jean-Louis Beffa, président du groupe Saint Gobain qui vient alors de racheter le groupe Poliet.

Après un peu plus d'un an de développement et un intense travail opérationnel, le premier dépôt ouvre, à Nanterre, le 5 janvier 1998. Très rapidement, d'autres s'installent à Nice (mai 98), Lyon (octobre 1998), Aubervilliers (avril 1999), Villemomble (octobre 1999). En 2002, on compte 17 dépôts 
en France et aujourd'hui plus de 60. Outre l'extension rapide du nombre de points de vente, le chiffre d'affaire témoigne de la bonne santé de l'enseigne, la plus rentable du groupe Saint-Gobain. Le succès de la Plateforme du bâtiment apparaît également dans les chiffres. La première année, le magasin de Nanterre affiche un chiffre d'affaire de plus de 55 millions de francs, moins de 10 plus tard il est de plus de 600 millions d'euros. Une success story qui dure encore et qui a valu à Gérard Aspar de recevoir, en 2003, les Arches de l'innovation, prix du groupe Saint-Gobain où domine pourtant fortement la culture technique !

Si le succès de cette innovation marketing n'est aujourd'hui plus à démontrer, se pose la question de comprendre quels sont les facteurs qui ont contribué à sa genèse.

Pour ce faire, nous commencerons par décrire les principales innovations de la Plateforme en les situant par rapport aux autres modèles de diffusion de matériaux. Dans un second temps, nous montrerons comment les contraintes de conception mettent le concept initial à l'épreuve et comment les concepteurs vont les contourner ou les intégrer. Enfin, la troisième partie tirera, dans l'exemple de la Plateforme du bâtiment, les éléments pouvant confirmer la présence de deux types de rationalités, créatives et analytiques, dans le processus d'innovation.

\section{La Plateforme du bâtiment : éléments de l'innovation}

Pour bien comprendre en quoi la plateforme du bâtiment est une innovation il est nécessaire de présenter les grandes de lignes de cette innovation et de les situer par rapports aux autres modèles de vente de matériaux qui existaient à l'époque.

\section{1. « $100 \%$ pro »}

Le caractère novateur de la Plateforme du bâtiment réside d'abord dans son positionnement puisqu'il s'agit de la première enseigne de distribution de matériaux professionnels généraliste exclusivement réservée aux professionnels. Contrairement aux GSB elle ne s'adresse pas aux particuliers, ces «bricoleurs du dimanche » qui n'ont ni les mêmes besoins ni les mêmes exigences qu'eux. «Les pro sont chez eux à la Plateforme », tel est le message construit par ses concepteurs pour les y attirer. Les produits qui y sont diffusés sont spécialement sélectionnés, il s'agit de marques professionnelles et c'est sur ce positionnement que la Plateforme a assis sa légitimité. Elle se démarque par ailleurs des circuits réservés aux professionnels de produits comme la peinture ou l'électricité qui sont mono-métiers.

\section{2. "Tout sous le même toit "}

La Plateforme du bâtiment s'adresse aux petits artisans urbains spécialisés dans la rénovation. Deux caractéristiques sont à prendre en compte. D'abord, ils sont concernés par l'ensemble des corps de métiers, et ont donc besoin de produits très différents, allant du gros œuvre (murs, charpente, etc.,) au second œuvre (électricité, peinture, etc.), en passant par l'outillage et la quincaillerie. Il faut donc une large gamme de produits. Ensuite, l'environnement urbain, très contraignant pour les trajets en utilitaires, les pousse à limiter leurs déplacements. La Plateforme du bâtiment a donc développé un concept existant pour le grand public, par exemple chez Home Depot, mais nouveau pour les professionnels, le «one stop shopping » leur permettant de réaliser leurs approvisionnements en une seule fois. Ainsi, et comme le souligne Maurice Manceau, [la Plateforme] «a été innovante à l'époque sur le fait de dire dans un même lieu je trouve tout ce que je veux pour faire de la réno, ce qui n'existait pas, même chez Point P. » [MAN 18]. Pour cela les concepteurs de la Plateforme ont imaginé une gamme de produits très large découpée en plusieurs espaces de plus petites plateformes libre-service : plombier, carreleur, peintre, menuisier, maçon, outillage, électricien. 


\section{3. « Le libre-service»}

Le libre-service est également une innovation dans le monde professionnel. Certes, c'est le principe de la GSB, mais, de nouveau, il faut souligner que les GSB, alors émergentes, ne captaient que peu de professionnels. La Plateforme se démarque du négoce en mettant le plus possible de produits en linéaire. L'artisan peut voir, toucher, se rendre compte par lui-même de ce qu'on lui propose avant de se servir ou de passer sa commande au guichet pour les produits les plus volumineux ou pour les quantités les plus importantes.

\section{4. «Des produits en stock»}

Autre élément novateur : l'assurance de trouver les produits en stock. Cette promesse, qui s'oppose au modèle du négoce, implique de suivre une gamme peu profonde pour éviter des stocks trop volumineux et donc une immobilisation de capital importante. Une innovation qui peut paraître risquée car elle est assortie d'une contrepartie : pas de contre-marque, c'est-à-dire, pas de commande possible. Les seuls produits disponibles sont ceux du catalogue, réputés disponibles dans tous les dépôts.

\section{5. «Des prix nets garantis et non négociables »}

La question des prix est également une ligne profonde de singularisation de la Plateforme par rapport au négoce dont la particularité est moins le prix du produit acheté que la remise que l'acheteur parvient à obtenir du vendeur (les prix affichés n'étant qu'indicatifs ou appliqués au tout petit artisan ou au particulier). La plateforme prend le contrepied de cette habitude en proposant un même prix pour tout le monde, affiché net et garanti. L'affichage, dans un catalogue, de prix non négociables constitue pour l'époque une offre «révolutionnaire » [MAN 18]. C'est une façon de poser deux grands principes : la transparence et une image de compétitivité. Si ce choix bouscule les habitudes, il devient un atout pour le petit professionnel qui pouvait s'estimer être le grand perdant du système de négoce :

«Ce sentiment d'équité rassure en fait l'artisan qui, s'il n'est pas bon négociateur, ne sait jamais s'il a eu les bons tarifs ou les bonnes conditions. Alors que là, c'était clair dès le départ [...] quand je suis maçon et que de temps en temps, j'ai besoin d'un peu de peinture... Je connais pas la peinture ! C'est pas mon métier, je n'ai pas les prix de référence, je connais pas les produits, etc. Quand je vois un peintre qui achète de la peinture [à la Plateforme] et que moi de temps en temps je dois acheter de la peinture, je suis rassuré parce que je vois des peintres, habillés en blanc, qui achètent de la peinture. Et je vais acheter le même prix que lui. » [VEN 18].

\subsection{Des services sélectionnés et combinés}

$\mathrm{Au}$ moment de la conception de la Plateforme du bâtiment, le négoce se caractérise par une très grande offre de services. Ceux-ci ne sont pas toujours formalisés : le client demande, le négociant s'efforce de répondre. De son côté, la GSB n'en propose presqu'aucun. La Plateforme a opté pour un positionnement médian : peu de services, mais des services ciblés vers l'artisan urbain spécialisé dans la rénovation. La location de matériel, grâce à un partenariat avec Kiloutou, a constitué un véritable argument en direction des petits artisans polyvalents qui ne peuvent investir dans du matériel trop spécifique. Quant à l'ajout d'une déchetterie à l'extérieur des magasins, cela a également constitué une innovation qui permettait aux artisans de gagner un trajet puisqu'ils déposent leurs déchets en allant faire leurs approvisionnements. "Là aussi, aujourd'hui c'est devenu un must, c'est quasiment une obligation légale ou presque. » [CAR 18]. La plateforme a enfin misé sur le conseil, dans les magasins eux-mêmes, et cela d'autant plus que la cible qu'elle cherche à attirer est composée de petits professionnels pas toujours bien formés : 
«Devant l'entrée des six secteurs du magasin (carrelage, plomberie, électricité, peinture, menuiserie, maçonnerie), un conseiller technique - spécialiste du domaine - est disponible. Présent en permanence dans son rayon, il est formé pour expliquer les méthodes de pose ou de montage des matériaux. "Le professionnel du bâtiment est devenu un généraliste qui jongle avec différents métiers, mais qui ne connaît bien que celui pour lequel il a été formé, explique Patrice Richard. D'où l'importance du conseil. » [S.N., 99]

Les dimensions novatrices de la plateforme ayant été présentées intéressons-nous à présent à la genèse de cette innovation.

\section{Genèse de la Plateforme du bâtiment}

\subsection{La Plateforme du bâtiment fruit d'une synthèse ingénieuse}

Un constat s'impose d'emblée : la Plateforme du bâtiment est une innovation de synthèse liée à l'importation et l'adaptation d'idées. Ce procédé a été clairement revendiqué par Gérard Aspar $^{1}$ et l'équipe originelle de la Plateforme du bâtiment comme une modalité à part entière de l'innovation. La Plateforme est effectivement décrite comme «Un concept professionnel moderne et innovant intégrant immédiatement les évolutions et les facteurs clés du succès existant chez d'autres : notamment dans les différentes formules de distribution dans notre environnement, mais également dans d'autres métiers ainsi que dans d'autres pays » [S.N., 97]. Un important travail de benchmark ${ }^{2}$ a ainsi été réalisé pour identifier et adapter des modèles qui fonctionnent, notamment en multipliant les voyages d'études :

«Donc dès les premiers mois, on est allé faire quelques voyages en Belgique un peu, aux Etats-Unis, au Canada, pour voir les modèles, le modèle étant Brico-Dépôt [en fait Home Depot]. Enfin Brico-dépôt façon US qui n'était pas un concept véritablement professionnel mais beaucoup plus orienté particuliers. Sur toute cette phase benchmark dans les premiers mois, je suis donc allé voir Brico-Dépôt [en fait Home Depot], au Canada Réno-Dépôt, pas mal de Leroy Merlin, Bauhaus en Allemagne, enfin tous ces concepts qui étaient un peu des concepts de distribution modernes. » [CAR 18].

Ce benchmark est mis en place pour l'ensemble des étapes de développement de la Plateforme du bâtiment. Très amont, entre 1994 et 1996, au moment même où se dessinent les premières idées d'un circuit alternatif de distribution de matériaux, le groupe de travail avait exploré différentes modalités de distribution. Le benchmark vise alors les modèles, par exemple la grande distribution, la vente par correspondance, etc. Dans la phase de développement plus opérationnelle, le benchmark cible des solutions plus concrètes permettant de mettre en œuvre tel ou tel principe, par exemple le gain de temps d'enlèvement des produits par les clients, la facilité d'accès aux produits exposés, etc., et/ou cherche, de façon beaucoup plus ouverte, toutes les idées qui pourraient s'adapter à la Plateforme du bâtiment et lui apporter un plus.

Le benchmark apparait ainsi comme une phase d'inspiration qui agit comme le terreau fertile de la phase d'idéation. Il ne s'agit toutefois pas uniquement de repérer une idée pour la décliner telle quelle en France ou dans un autre secteur. Tout se joue dans la capacité de Gérard Aspar et son équipe à créer, à partir de cette matière existante, une offre innovante :

\footnotetext{
${ }^{1}$ Ce procédé avait d'ailleurs été déjà en partie appliqué aux magasins Lapeyre lorsque Gérard Aspar était directeur général de l'enseigne.

${ }^{2}$ Le benchmark est une technique de marketing centrée sur l'observation et l'analyse des pratiques concurrentes en vue de s'en inspirer et d'en tirer le meilleur.
} 
«Objectif : reprendre le meilleur de chacun des grands concepts qui font référence dans la distribution et même dans la grande distribution, qu'ils se soient développés dans d'autres métiers que celui du bâtiment ou dans d'autres pays. « Nous avons beaucoup retenu des leçons de distributeurs comme Ikea (décoration, mobilier), Castorama (grande surface de bricolage), Home Dépot (matériaux de construction aux Etat-Unis), Toys R’Us (jouets), Darty (électroménager, Métro (centrale d'achat pour l'alimentation), Auchan (Hypermarché), etc. » détaille Gérard Aspar. » [S.N., 98].

De fait, la liste des emprunts est longue :

- METRO : La Plateforme a emprunté le principe du cash \& carry de cette enseigne. Elle a aussi élaboré un système de filtre, importé de chez METRO : la carte d'accès. Délivrée sur présentation du K-bis (document officiel attestant de l'existence juridique de l'entreprise et de son inscription au Registre du Commerce et des Sociétés), cette carte d'accès agit comme une carte de membres et renforce l'impression d'un espace privilégié, réservé aux professionnels ;

- Brico Dépot: le remboursement du prix si le client trouve moins cher ailleurs, qui installe l'image d'un positionnement de prix bas ;

- Carrefour : l'organisation des rayons avec une vision univers / métiers permettant d'organiser des magasins immenses puisque rassemblant « tout sous le même toit »;

- Grands magasins : les "corners marque", soit les espaces de vente dédiés à une marque en particulier à l'intérieur des magasins, offrant de la visibilité aux marques professionnelles dont la présence est rassurante pour le professionnel qui les connaît

- Office Depot : les zones basses au centre ;

- Home Depot USA : le business model prix bas, couleur orange ;

- Contractor's Warehouse USA : les horaires d'ouverture très tôt le matin. «Faire gagner du temps et de l'argent» aux artisans a servi de base ligne à la Plateforme du bâtiment et à l'ouverture à $6 \mathrm{~h} 30$ en anticipant les besoins d'un artisan urbain qui passe une bonne partie de son temps dans les bouchons!

- Magnet Southern UK : le pacte de remboursement si rupture de stock ;

- Michelin : le demi format type guide vert pour le catalogue des prix permettant de l'avoir dans la boite à gants ou dans la poche ;
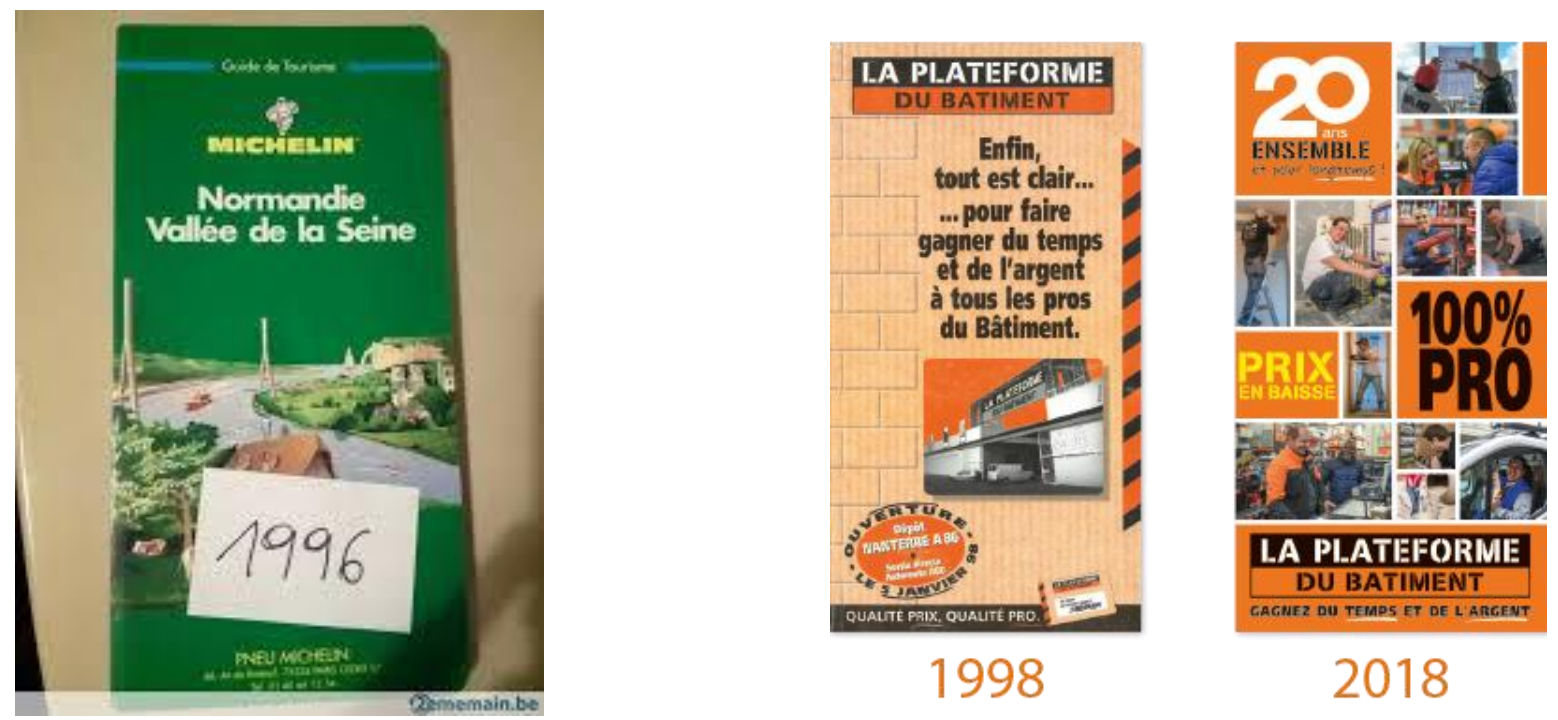
- FNAC : le format des sacs plastiques ;

- Quai des marques : les bannières verticales et marques en façade

- Lapeyre : le drive in ;

- B \& Q : la cafétéria, renforçant l'effet de club et d'attention portée aux artisans ;

- etc.,

\subsection{La conception comme processus de gestion de contraintes hétérogènes}

\subsubsection{Les contraintes de quoi parle-t-on?}

L'histoire de la genèse de la Plateforme du bâtiment réactualise la question du rôle des contraintes dans l'innovation. On tend en effet à envisager a priori la relation de l'innovation aux contraintes en termes de contradiction. Ce point de vue est cependant largement contesté à la fois des scientifiques de renom à l'instar d'Einstein selon qui chacun agit sous une contrainte extérieure et d'artistes comme l'illustre l'exemple du roman La Disparition (1969) de Georges Perec qui ne comporte pas la lettre $e$. L'innovation semble ne pas échapper à cette règle car, quiconque s'est trouvé un jour en situation de conception sait que le processus de conception est par nature un processus de gestion de contraintes hétérogènes et qu'il appartient au concepteur de contourner, intégrer ou repousser les contraintes de la situation dans laquelle il agit ${ }^{3}$. Quatre types de contraintes peuvent ainsi être distingués [FOR 17] :

- les contraintes liées à la définition du problème, à la fois posées par l'utilisateur (facilité d'utilisation), l'entreprise (contrainte de marché, de viabilité...) et le concepteur (Le développement d'innovations qui répondent aux préceptes du développement durable s'opère dans une société où la question de notre responsabilité vis-à-vis de la planète et des générations futures est prégnante).

- les contraintes liées à l'environnement de la conception et sont révélées par le concepteur. Spontanément on pense ici aux normes de produit en vigueur (norme NF par exemple), aux contraintes légales (à l'instar de l'interdiction de faire de la publicité pour le tabac) et aux contraintes environnementales (c'est par exemple l'obligation pour les fabricants d'équipements électriques et électroniques de prendre en compte la directive 2002/95/CE du 27 janvier 2003 relative aux substances dangereuses contenues dans ces équipements et la directive 2002/96/CE du 27 janvier 2003 relative aux déchets d'équipements électriques et électroniques). Entrent aussi dans cette catégorie les limites des connaissances scientifiques et techniques.

- les contraintes héritées et propagées des phases amont du processus de conception (le choix à une étape de pouvoir désassembler le produit se propage à l'étape suivante comme une contrainte qui conduira à privilégier la modularité).

\footnotetext{
${ }^{3}$ En d'autres termes l'action du concepteur est l'expression (et la mise en œuvre) d'une liberté dans le cadre dudit environnement ce qui revient à souligner que c'est le subtil mariage des contraintes et la liberté du concepteur qui modèlent l'artefact final.
} 
- les contraintes qui émanent des acteurs du processus de conception que l'on fasse ici référence au problème de la rationalité limitée de l'acteur économique évoqué par H. Simon [SIM 57] où à leur appréciation de la situation [FAL 90].

Si l'on admet l'idée selon laquelle une multiplicité et hétérogénéité de contraintes pèsent sur le processus de conception, toutes ne sont pas de même nature. Considérer les contraintes inhérentes à tout processus de conception permet en outre de comprendre que le concept final ne fait que révéler ou traduire les compromis réalisés au cours du processus de conception, comme nous allons le voir à partir du cas de la Plateforme du bâtiment.

\subsubsection{Quelles contraintes dans le cas de la Plateforme?}

\subsubsection{Les contraintes liées à l'environnement de la conception}

La contrainte externe majeure qui s'est imposée à la Plateforme est la promulgation de la loi Raffarin. En 1996, le projet tel qu'il s'esquisse est un concept de cash and carry destiné au grand public et aux petits professionnels, calqué sur le modèle de Home Depot, une enseigne américaine créée à Los Angeles en 1978. Or, durant l'été 1996, la loi n 96-603 relative au développement et à la promotion du commerce et de l'artisanat, dite "loi Raffarin" impose aux grandes surfaces de plus de $300 \mathrm{~m}^{2}$ d'obtenir une autorisation par une Commission départementale d'équipement commercial (CDEC). Avec cette loi, «On va être sur des temps de plusieurs années alors que nous on voulait développer ça sur moins de deux ans. » [CAR 18]. Gérard Aspar contourne cette contrainte très rapidement et de manière "opportuniste" en faisant le choix de faire évoluer le concept et de le réserver aux seuls professionnels. Avec le recul, Gérard Aspar précise :

«Ça m'a aidé à faire quelque chose de beaucoup plus pur, alors que je voulais faire un truc aux pros et particuliers, si je suis 100\% comme METRO, METRO n'est pas soumis à la fameuse CDAC autorisation grandes surfaces » [ASP 17].

Sans aucun doute, cette réorientation du concept s'avérera l'élément le plus distinctif de la Plateforme du bâtiment. C'est lui qui fait véritablement rupture avec les modes de distribution précédents qui étaient tous ouverts aux professionnels et aux particuliers, à tel point que Patrick Venzal dit que « la loi Raffarin qui était une contrainte [...] est devenue un avantage énorme » [VEN 18].

Ce changement d'orientation met en avant l'inscription sociale des innovations. Ces dernières se développent dans un environnement qui définit le champ des possibles et à l'intérieur duquel le concepteur va pouvoir «jouer» sa propre partition. La loi conduit ainsi à faire bifurquer le concept dans une nouvelle direction qui sera validé plus tard par des études.

\subsubsection{Les contraintes héritées et propagées des phases amont du processus de conception}

Comme nous l'avons indiqué, le public visé n'est pas celui qui avait été initialement envisagé par les concepteurs, qui incluaient le grand public. Cette bifurcation conduit à affiner le concept en analysant les besoins des futurs usagers de la Plateforme car, comme on le sait, une innovation implique de créer de la valeur pour les usagers. Or, consécutivement à la réorientation du projet, Gérard Aspar et son équipe s'aperçoivent de la transformation du métier d'artisans. Ils formalisent un concept de profil émergeant : le JAUM, «le Jeune Artisan Urbain Moderne » [CAR 18]. Il est vu comme un petit artisan un peu précaire, spécialisé dans la rénovation et donc polyvalent, qui ne

\footnotetext{
${ }^{4}$ Son concept de rationalité limitée précise les limites du décideur confronté à un problème de décision. Il s'agit en l'occurrence : d'une connaissance imparfaite ou bornée de son environnement, de l'impossibilité d'anticiper et de considérer toutes les options relatives au problème posé du fait de ses capacités de calcul finies, de l'impossibilité de traiter toutes les informations qui se présentent du fait des limitations de son attention. La décision obéit ainsi au principe du satisficing [FOR 01].
} 
possède pas une grande surface financière et maîtrise mal les outils de la négociation, surtout lorsqu'il est étranger.

« Il y a un type d'artisans qui apparaissait sur l'Ile-de-France qui était multi-métiers avec une surface financière assez réduite, c'est-à-dire pas forcément susceptible d'avoir du crédit, en tout cas au démarrage, $[\ldots]$, mais payant cash, très cash même, $[\ldots]$ orientés vers la rénovation. » [CAR 18].

Cet artisan, qui fait peu de volume, intéresse peu le négoce qui ne lui offre pas les meilleurs conditions d'achat. De ce fait, le «petit pro » se sent peu considéré par ce format de vente. Ainsi, en se cherchant un nouveau modèle, la Plateforme va rapidement identifier ce nouveau public-cible qui a besoin de reconnaissance :

«Ce petit pro est mal traité partout parce qu'il faut qu'il achète un peu de choses chez Point P, chez Electricité Machin, chez Peinture Bidule, et chez je ne sais pas quoi Matériaux, et donc il est petit tous les jours, il fait deux heures de courses et on le prend pour un con parce qu'il est tout petit, il n'a pas les bons prix, il n'est pas considéré » [RIC $18]$.

"Quand vous êtes dans le négoce, les petits artisans, les petits, grosso modo on ne s'en occupait pas. Et c'est normal. Quand vous êtes dans la distribution de produits pour le bâtiment, plus vous êtes gros plus on s'occupe de vous, et a contrario plus vous êtes petit et moins on s'occupe de vous ${ }^{5}$. [...] J'ai dit, moi, c'est très bien, ils ont leur métier et c'est parfait, c'est un super modèle d'avoir un négoce avec des remises, des services, des comptes clients, des showrooms, des gens qui vendent à l'extérieur. Parfait! Mais moi je veux être un concept où au contraire je ne veux m'occuper que des petits. Les gros, à la limite, je n'en veux pas! Donc je vais faire tout le contraire, je vais m'occuper, me focaliser sur l'insatisfaction des petits et le petit, qu'est-ce qu'il veut? Il veut une offre simple, courte, en stock, point c'est tout, de qualité bonne, sans réfléchir. Donc quelque part ça a été de dire je ne vais faire qu'une seule offre, ultra simple, avec des prix, prêt à emporter, près de chez eux. C'est là où quelque part dans les insatisfactions, et ça a été la clef de voûte un peu de la promesse client » [ASP 17].

Parallèlement, à partir de 1997, et jusqu'en 2008, la période est économiquement favorable pour la construction $^{6}$ et les demandes en rénovation croissent dans les grandes villes. Cette conjonction de facteurs crée des conditions porteuses pour la Plateforme du bâtiment, conditions qui n'avaient pas été totalement anticipées :

« Honnêtement, je pense que ça a été un coup de chance d'être là au bon moment, au bon endroit, sur une demande et un marché qui avaient des besoins et une rénovation qui explosait. Faut avoir un peu de chance aussi ! » [VEN 18].

De ce qui précède, il ressort que loi Raffarin, parce qu'elle a contraint l'équipe faire un choix et à cibler les professionnels, a conduit à mener une étude sur leur profil et leurs besoins afin de spécifier la cible visée : "Ils ont trouvé le concept et après ils ont trouvé auprès de qui ça marchait et après, ça a

\footnotetext{
5 La critique de la distinction entre les gros clients et les petits clients opérée par le négoce fut un argument fort des campagnes de communication de la Plateforme du bâtiment. Présente dès le premier catalogue, elle fut pour beaucoup dans les tensions entre le Plateforme et Point P. «Finis le mélange professionnels et particuliers, les prix à la tête du client, les marques sans garanties, les fausses promotions, les produits non disponibles, les journées passées d'un grossiste à l'autre... " (Dans le $1^{\mathrm{er}}$ Memento de la Plateforme du bâtiment [S.N 98]).

${ }^{6}$ Selon la Fédération Française du Bâtiment, le secteur aurait créé plus de 320000 emplois en 10 ans [FED 11]. Voir aussi [PER 15].
} 
guidé les stratégies d'implantation, de développement, etc.» [BEF 18]. Comme nous allons le voir, le positionnement retenu va conduire Gérard Aspar et son équipe à définir des principes de solution qui vont eux même agir comme des contraintes au niveau opérationnel. De fait, plusieurs des contraintes qui désignent l'offre Plateforme sont directement héritées de cette nouvelle cible : des prix affichés, des produits visibles, du conseil ciblé sur les produit, etc.

\subsubsection{Les contraintes liées à la définition du problème posées par le concepteur}

Les concepteurs de la Plateforme du bâtiment sont à l'origine de contraintes qui n'ont pas été déterminées au hasard mais résultent de choix faits, pour des raisons multiples, qui vont de la sécurisation des revenus de l'entreprise à l'appropriation du concept par la cible visée. Gérard Aspar et son équipe sont en effet à l'origine de ce qu'ils qualifient de «choix tranchés», pour proposer «une offre claire », "pure », là encore inspirés d'autres expériences, comme celles de McDo, Ikéa, Zara [ASP 17]. Il faut ici entendre que, dès le départ, les concepteurs ont posé franchement la distinction entre «Ce qu'on fait» / «Ce qu'on ne fait pas ». Ce principe vaut dès 1996, mais la loi Raffarin a obligé les concepteurs à être encore plus «purs» en renonçant à la cible des particuliers. Après avoir identifié clairement les usagers visés, l'idée d'une offre claire se matérialise dans un certain nombre de contraintes définies par Gérard Aspar et son équipe : un accès en libre-service, la règle des $3 \times 20$ minutes ${ }^{7}$, pas de crédit, des prix fixes et non négociables, tout en stock, pas de livraison.

Ces choix agissent à leur tour comme des contraintes opérationnelles qu'il faut prendre en compte dans le passage du "concept" Plateforme à la réalité des magasins. Par exemple, le libre-service implique de penser les linéaires en intégrant la question du stockage des produits, celle de leur mise en valeur, de l'affichage des prix. Il fallait «inventer un merchandising qui parle aux clients... sortant $d u$ négoce » [VEN 18].

La règle des 3 x 20 minutes s'est traduite par une stratégie d'implantations finement maillées, avec des horaires d'ouverture tôt permettant d'éviter les embouteillages, complété par un travail sur la présentation des produits dans les linéaires, leurs disponibilités et les délais d'enlèvement. Cet argument a été l'un des plus forts, jusqu'à servir de base line commerciale, puisque, pour Gérard Aspar, «tout mon concept, ça va être de gagner du temps et gagner de l'argent, et dans cet ordre-là. Je vais tout faire pour gagner du temps. Et ça a été fondamental! [...] On a fait une révolution » [ASP 17].

Si tous les choix faits par les concepteurs leur fournissent un système de contraintes qu'ils s'efforcent d'intégrer dans la phase de développement opérationnel, certaines seront difficiles voire impossibles à tenir et vont nécessiter des compromis. Dans le cas du crédit et des livraisons, par exemple, il apparait que tenir la contrainte serait contreproductif vis-à-vis de la cible.

Concernant le crédit. Gérard Aspar et Patrice Richard citent une étude de marché réalisée, probablement fin 1996 début 1997, pour tester le concept de la Plateforme. Elle indiquait que $80 \%$ des artisans interrogés ne s'y rendraient pas. En cause ? Essentiellement l'impossibilité d'y avoir un compte. Dans ce cas précis, la contrainte énoncée par le concepteur porte un risque pour la viabilité du projet. En effet, il faut avoir à l'esprit qu'un artisan dans le bâtiment n'est payé par le client pour le compte duquel il réalise des travaux qu'à la fin du chantier. Aussi le négoce jouait le rôle du banquier ce qui arrangeait d'autant plus ces petits artisans qui, comme nous l'avons indiqué précédemment, disposaient d'une faible surface financière. A l'idée de «ne pas faire de crédit » s'est substituée celle de «ne pas jouer le rôle de banquier» ce qui, au niveau opérationnel, a conduit à retenir comme solution de recourir à un service externalisé de crédit. La différence de formulation de cette contrainte

\footnotetext{
7 «20 minutes au maximum pour que l'artisan vienne, 20 minutes pour qu'il fasse ses achats et 20 minutes pour qu'il retourne sur son chantier $\gg[$ S.N 08a].
} 
peut paraître triviale mais il n'en est rien car au final elle permet à Gérard Aspar et son équipe d'exprimer cette contrainte en termes de fonction attendue «la Plateforme n'avance pas d'argent et n'assume pas le risque d'un défaut de paiement » et non de solution «pas de crédit». Or cette seconde formulation conduit à explorer de nouvelles solutions à proposer au client leur permettant d'étaler leurs paiements et de (presque) retrouver la souplesse dont ils disposaient dans le négoce :

« Pour les comptes, on n'a pas la solution au départ. Mais très vite on se rend compte qu'on en a besoin et là c'est un truc un peu à part, alors autant Point $\mathrm{P}$... la force de Point $\mathrm{P}$ c'est d'être son propre assureur crédit et de gérer ses comptes avec des gens... autant là, on fait appel à une banque, la SEDEF avec une carte spéciale, une organisation spéciale, un montant. Mais ça fonctionne. Ça fonctionne, mais je vous dis ça pèse $10 \%$ » [VEN 18].

Pour ce qui concerne la livraison, la contrainte a été partiellement levée très rapidement, fin 1998, pour pouvoir accéder au marché du gros œuvre :

«En fait on s'aperçoit que le gros œuvre ça se livre et que c'est un atout qu'il faut avoir sinon on n'aura pas le gros œuvre. La volonté de la Plateforme d'être le premier second fournisseur des artisans, pour certains on devient le premier fournisseur. Et puis on pense qu'on fera que de la réno mais on fait aussi un peu de neuf et puis des fois il y a des grosses réno, il faut livrer donc la livraison c'est un camion, puis deux camions, et on commence à livrer. [...] Quasiment, sur Nanterre, très vite et après sur toutes les Plateforme il y avait un camion. Sur toutes les Plateforme, très vite, très très vite, on se rend compte [...] qu'il faut livrer. » [VEN 18].

\subsubsection{La contrainte du groupe : entre rejet et soutien, frein et opportunité}

À l'intérieur d'un grand groupe, l'innovation est souvent considérée comme un moteur nécessaire de croissance en même temps qu'elle est difficile à faire émerger. Pour expliquer cette tension entre d'une part le besoin de se transformer et, d'autre part, le renfermement sur soi-même qui fait frein à cette transformation, Jean-Louis Beffa reprend la notion de «dilemme de l'innovateur» de Clayton Christensen: "L'acteur installé repousse les innovations trop radicales car il poursuit un but, satisfaire en permanence et efficacement la demande de ses consommateurs. Pour lui, la position dominante devient un frein à l'innovation. Il a trop à perdre à prendre le risque de surprendre ses clients. La start-up est l'agent de la rupture car elle n'a rien à perdre » [BEF 17, p. 29-30]. Si l'innovation porte en elle les germes de la destruction des modèles et des structures qui la précède, alors on comprend aisément que ceux-ci cherchent à se protéger.

Cette dimension est effectivement présente dans l'exemple de la Plateforme et se manifeste en particulier par l'affrontement de deux modèles de diffusion de matériaux concurrents : celui de la plateforme et celui du négoce incarné par Point P. Elle sera notamment attisée par l'éditorial du premier mémento de la plateforme à l'occasion de l'inauguration de la plateforme. On a déjà dit que la Plateforme était une offre alternative au négoce et qu'elle cherchait à en dépasser ce qu'elle percevait comme des faiblesses, on n'a pas encore souligné à quel point l'exploitation de cette position avait été forte, voire agressive : pour faire la promotion du modèle Plateforme, Gérard Aspar insiste sur les failles du négoces sans jamais en évoquer les atouts. Le jeu est cruel, sans doute un peu facile, mais assez naturel dans la concurrence que se livrent les enseignes. Mais l'appartenance de celles-ci au même groupe rend insupportable l'assaut qui est ressenti comme une insulte par le négoce et ceux qui le pratiquaient. Avec le recul, Gérard Aspar juge la charge lourde mais il «l'assume », car il «disai[t] tout haut ce qui était la vérité » [ASP 18]. 


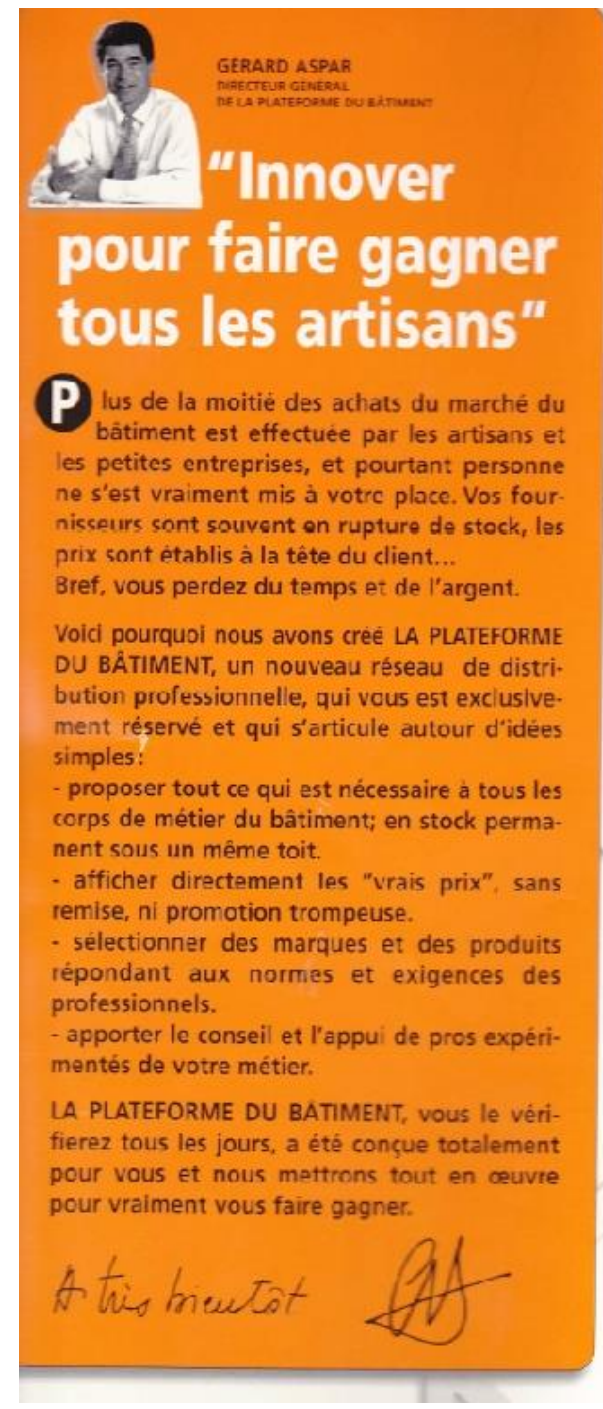

"Finis le mélange professionnels et particuliers

les prix à la tête du client, les marques sans

garantie, les fausses promotions, les produits

non disponibles, les journées passées

d'un grossiste à l'autre..."

\section{Chez nous,}

tout est clair...

...pour vous

faire gagner

du temps

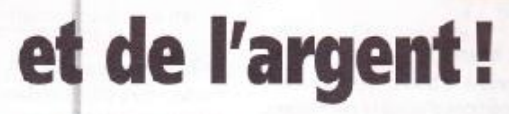

Et nous nous engageons

avec le PACTE PRO.

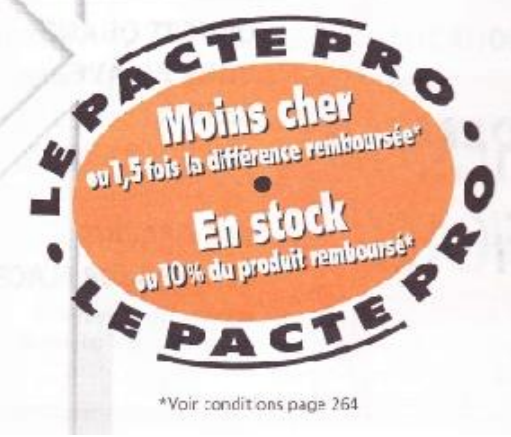

Figure 1. Édito du premier mémento de la Plateforme du bâtiment, 1998 ([S.N 98])

D'où qu'elles viennent, les contraintes imposent au concept initial de s'adapter sous peine de ne pas répondre aux impératifs de faisabilité, de mal répondre aux attentes des cibles visées, de manquer les enjeux internes à l'entreprise, etc., Ce constat permet de s'émanciper de l'idée qu'il suffirait de faire émerger un concept pour innover. Encore faut-il qu'il passe l'épreuve des contraintes. L'issue de cette confrontation dépend de la capacité des concepteurs à trouver des solutions pour intégrer ou contourner les différentes contraintes qui émergent au cours du processus de conception.

Chemin faisant, l'exemple de la Plateforme du bâtiment révèle que l'innovation résulte du déploiement deux types de rationalité : la rationalité créative et la rationalité analytique.

\section{Rationalité créative et analytique : deux formes de pensée nécessaires pour innover}

\subsection{L'histoire de la plateforme conforte le rôle de la rationalité créative dans l'innovation}

Dans Creative rationality and innovation nous avons indiqué que la rationalité créative est à l'origine même de l'innovation. Proche du concept de bissociation [KOE 64], la rationalité créative s'apparente à une pensée de la relation qui, en combinant des connaissances, en produit de nouvelles à l'origine même de l'innovation. Le déploiement de cette forme de pensée invite à la traversée des savoirs, à aller voir en dehors de sa discipline ou de son secteur car le spectre de l'expansion sera d'autant plus grand que les connaissances seront variées [FOR 17]. Ainsi conçu, le déploiement de la rationalité créative suppose de posséder les «gènes de l'innovateur » [CHR 13] et en particulier de posséder : un sens aigu de l'observation, un esprit ouvert, curieux et une appétence pour la rencontre, l'altérité. 
Ce sont précisément des traits de caractère que l'on retrouve chez Gérard Aspar qui possède une forte capacité d'observation et, pourrait-on dire, d'imprégnation. À plusieurs reprises, dans les deux entretiens qu'il nous a accordé, ressortent les expressions «j'avais $v u », ~ « j e ~ c o n n a i s s a i s »{ }^{8}$. Elles témoignent de l'ouverture d'esprit et de la curiosité d'un homme qui cherche en permanence à se nourrir en observant d'autres expériences et en questionnant des personnes qui le conduise à faire des liens et à se définir un peu comme un japonais, selon le cliché qui veut que le modèle japonais d'innovation procède par emprunts et duplication ${ }^{9}$.

«Mais il y avait un côté japonais, je veux dire il y a beaucoup de choses que j'ai prises à... c'est fait de micro innovations » [ASP 17].

Autant de qualités qui lui permettent de faire des liens et de combiner les savoirs et les univers. Le déploiement de la rationalité créative implique d'être porté par un leader engagé porteur d'une vision :

«Le bonhomme quelque part qui dégage une énergie phénoménale, qui a un projet qui est à la fois très clair et fou, qui consiste à prendre tout le dispositif, tout le business tel qu'il était installé, ce qu'on appelle le négoce en France, ce métier traditionnel assez peu structuré, de le prendre complètement de biais et faire la démonstration que ça peut apporter quelque chose de mieux aux clients, de plus pour l'actionnaire, de mieux pour les collaborateurs qui vont inventer un truc qui est différent et mieux. Voilà, la perspective de créer quelque chose de beau, légèrement utopique, sans doute un petit peu idéal et quelque part un peu absolu. Voilà !» [RIC 18].

Détermination d'autant plus nécessaire que la rationalité créative opère selon le registre de la transgression aventureuse et invite à se défaire des normes et paradigmes établis : «On nous avait dit : «ça ne marchera jamais. Jamais un artisan du bâtiment prendra son petit panier ou son chariot pour faire ses courses » [JOU 08a] et qui se fait toujours, au moins momentanément, contre l'ordre, même si elle finit souvent par participer à une autre conception de l'ordre [ALT 00], elle se heurte au conservatisme ambiant. Conservatisme qui conduira Gérard Aspar à «jouer» sur les résultats de certaines études pour éviter d'exposer le projet à d'éventuels refus. Ainsi explique Gérard Aspar,

« cette étude je l'ai un peu masquée... j'en ai parlé quelques années après, j'avais vu le président de Saint-Gobain que je connaissais très bien : "Il faut que je te montre quelque chose que je ne l'ai jamais montré", il me dit: "T'as bien fait de ne pas le montrer parce qu'on t'aurait arrêté le projet." » [ASP 17].

Soulignons toutefois que la possibilité d'effectuer cette transgression aventureuse n'est pas étrangère à un environnement qui la rend possible. Le projet a en effet été porté par Jean Louis Beffa, alors président du groupe, qui a également soutenu son indépendance (fonctionnement en mode start up) en vue de le protéger de l'inertie du groupe incompatible avec la réactivité nécessaire pour

8 «J'avais vu l'idée aux Etats-Unis », «J'avais vu se développer en Angleterre », «je connaissais quelqu'un » [ASP 18], «J'avais vu déjà chez Lapeyre », «J'avais vue au Chili », «J'avais vu, je me souviens très bien, du côté de Cincinnati », «J'avais vu les gens de Costcool », «Je connaissais énormément de gens vraiment dans beaucoup de monde. Je connaissais le patron stratégique de Home Dépôt (...) c'est vrai que j'avais bourlingué dans le monde de la distribution en France et à l'étranger, ce qui m'avait permis de me faire un certain nombre de convictions et d'être solide », « je connaissais très bien le patron fondateur de Brico Dépôt », «Les gens de Métro que je connaissais à l'époque, c'était... avant qu'il ne devienne président de Carrefour... [inaudible] qui m'avait fait rencontrer son directeur des ventes » [ASP 17].

9 Il faut aussi noter que cette capacité à faire des liens est partagée par l'équipe recrutée. Car si le benchmark, c'est-à-dire l'ouverture d'esprit à des expériences conduites dans d'autres secteurs que la construction ou dans d'autres pays est encouragé, le profil des personnes recrutées témoigne d'un même souci. Certains viennent du négoce ou de la GSB, d'autres de la grande distribution, d'autres encore de l'automobile. Chacun peut ainsi apporter un regard et une expérience différente. 
développer une innovation ${ }^{10}$. Le mode start up permet au projet une réactivité (agilité diraient certains aujourd'hui) au sein d'un groupe dont la taille a conduit, au nom de l'efficacité, à mettre en place une organisation, dont on sait qu'elle n'est pas toujours favorable à l'innovation [FOR 18].

Si la rationalité créative est à l'origine même de l'innovation, car elle ouvre le champ des possibles, elle cohabite avec la rationalité analytique dont la principale fonction est, comme nous allons le voir dans ce qui suit, de tester le concept imaginé ou, pour le dire autrement, de vérifier que les croisements de savoirs opèrent effectivement.

\subsection{La rationalité analytique comme modalité de validation de la rationalité créative}

Si l'exemple de la Plateforme du bâtiment fait ressortir l'importance de la rationalité créative dans sa genèse, celle-ci ne saurait suffire à rendre compte de la totalité du processus d'innovation. L'innovation repose également sur des phases où se déploie la rationalité analytique :

«Oui beaucoup d'instinctif, oui beaucoup de créativité, oui beaucoup de recherche au départ mais à chaque fois de se dire quand quelque chose marche, comment le dupliquer, comment l'améliorer, etc. Après il y a de l'analytique derrière, il y a beaucoup d'analytique. » [VEN 18].

De même, Gérard Aspar insiste sur cette dimension de rigueur, en soulignant combien «tout ça était très méthodique» [ASP 17]. À l'appui de cette idée, il produit le découpage préparatoire au développement de la Plateforme en 27 grands chantiers (illustrations ci-dessous).

«J'avais demandé énormément de méthodes, analytiques [inaudible]. C'est vrai que j'avais été très méthodique dans le travail, dans la préparation : "on va faire ça". » [ASP 18].

${ }^{10}$ Et dont nous avons vu qu'elle peut venir de l'intérieur même du groupe d'entité qui craigne pour leur pérennité. 


\begin{tabular}{|c|c|c|c|c|c|c|c|}
\hline CHANTIERS & PILOTE & $\begin{array}{c}\text { DEGRE } \\
\text { IMPORTANCE }\end{array}$ & $\begin{array}{l}\text { DEGRE } \\
\text { URGENC }\end{array}$ & & $\begin{array}{l}\text { DEGRE } \\
\text { INTERDEPENDANCE }\end{array}$ & $\begin{array}{l}\text { DEGRE } \\
\text { INTERN: }\end{array}$ & $\begin{array}{l}\text { XPERTISE } \\
\text { ERNE }\end{array}$ \\
\hline 1. ANALYSE CLIENTELES PAR CORPS DE METIERS & $\mathrm{BC}$ & $* \quad * \quad *$ & $\square \quad \square$ & $\square$ & 3 & $\Rightarrow$ & $\leftarrow$ \\
\hline 2. ANALYSE CLIENTELES SOCIOPROFESSIONNELLES & $\mathrm{BC}$ & $*$ & $\square$ & & 1 & $\rightarrow$ & $\leftarrow$ \\
\hline 3. CLIENTELE FINALE DE L'ARTISAN & $G A+D G$ & $* * *$ & $\square$ & & 1 & & $\leftarrow$ \\
\hline 4 CONSTITUTION DE GAMMES & BC & * * & $\square$ & & 3 & $\rightarrow$ & $\leftarrow$ \\
\hline 5. POLITIQUE MARQUES PROFESSIONNELLES & $\mathrm{BC}$ & $* \quad *$ & $\square$ & & 2 & $\rightarrow$ & $\leftarrow$ \\
\hline 6. POLITIQUE GENERALE ACHATS & $B C+G A$ & $* \quad *$ & $\square$ & $\square$ & 1 & & $\leftarrow$ \\
\hline 7. REFERENCEMENT DES GAMMES & $B C$ & $* \quad *$ & $\square$ & & 2 & $\rightarrow$ & \\
\hline 8. ETUDES SERVICES & $G A+B C$ & $* \quad *$ & 口 & & 1 & $\rightarrow$ & $\leftarrow$ \\
\hline 9. CIRCUIT DEPOT CLIENTS / PRODUITS & $P R+D G$ & * & 口 & & 3 & $\rightarrow$ & \\
\hline 10. SECURITE BIENS / PERSONNES & $\mathrm{DG}+\mathrm{PR}$ & $* \quad *$ & 口 & & 1 & $\rightarrow$ & \\
\hline 11. POLITIQUE COMMERCIALE ET MODES VENTES & $B C+P R$ & * * & 口 & & 2 & $\rightarrow$ & \\
\hline 12. MERCHANDISING ET IDENTITE VISUELLE & $B C+P R$ & * & ( 口 & & 2 & & $\leftarrow$ \\
\hline 13. EQUIPE DEPOT : PRINCIPES MANAGEMENT & $P R+G A$ & $* \quad * \quad *$ & $\square$ & & 1 & & \\
\hline 14. RECRUTEMENT EQUIPE NANTERRE & PR & * * & $\square$ & & 1 & $\rightarrow$ & $\leftarrow$ \\
\hline 15. RECRUTEMENT EQUIPE NICE & $G A+P R$ & $* *$ & $\square$ & & 1 & $\rightarrow$ & $\leftarrow$ \\
\hline 16. SYSTEMES D'INFORMATION & DG & $* *$ & 口 & & 3 & $\rightarrow$ & $\leftarrow$ \\
\hline 17. LOGISTIQUE / APPRO & $D G+P R$ & $*$ & 口 & & 2 & $\rightarrow$ & $\leftarrow$ \\
\hline 18. FINANCEMENT CLIENT & $P R+D G$ & * * * * & $\square$ & & 1 & $\rightarrow$ & $\leftarrow$ \\
\hline 19. BENCHMARKING AUTRES DISTRIBUTIONS & GA & * * & $\square$ & & 1 & & $\leftarrow$ \\
\hline 20. COMMUNICATIONS ET OUTILS & $B C+G A$ & $* *$ & $\mathrm{Q}$ & & 2 & $\rightarrow$ & $\leftarrow$ \\
\hline 21. RECHERCHES IMMOBILIERES & GA & * * & $\square$ & $\square$ & 1 & $\rightarrow$ & $\leftarrow$ \\
\hline 22. ETUDES MARCHES LOCAUX & $P R+B C$ & $* \quad *$ & 口 & & 1 & $\rightarrow$ & \\
\hline 23. LANCEMENT DEPOT 1 & $\mathrm{GA}+\mathrm{JFM}+\mathrm{PR}$ & $* *$ & $\square$ & & 2 & $\rightarrow$ & $\leftarrow$ \\
\hline 24. LANCEMENT DEPOT 2 & $\mathrm{GA}+\mathrm{JFM}$ & $* *$ & $\square$ & & 2 & $\rightarrow$ & $\leftarrow$ \\
\hline 25. INVESTISSEMENTS ET COUTS D'EXPLOITATION & $D G+G A$ & $* \quad * \quad *$ & 口 & & 3 & $\rightarrow$ & \\
\hline 26. SIIMULATIONS FINANCIERES / IMPACT PROJET & $G A+D G$ & $* \quad *$ & $\square$ & & 3 & & \\
\hline 27. ORGANISATION GALE / COORDINATION GROUPE & GA & $* * *$ & N.S. & & 1 & & \\
\hline
\end{tabular}

PLANNING GENERAL,

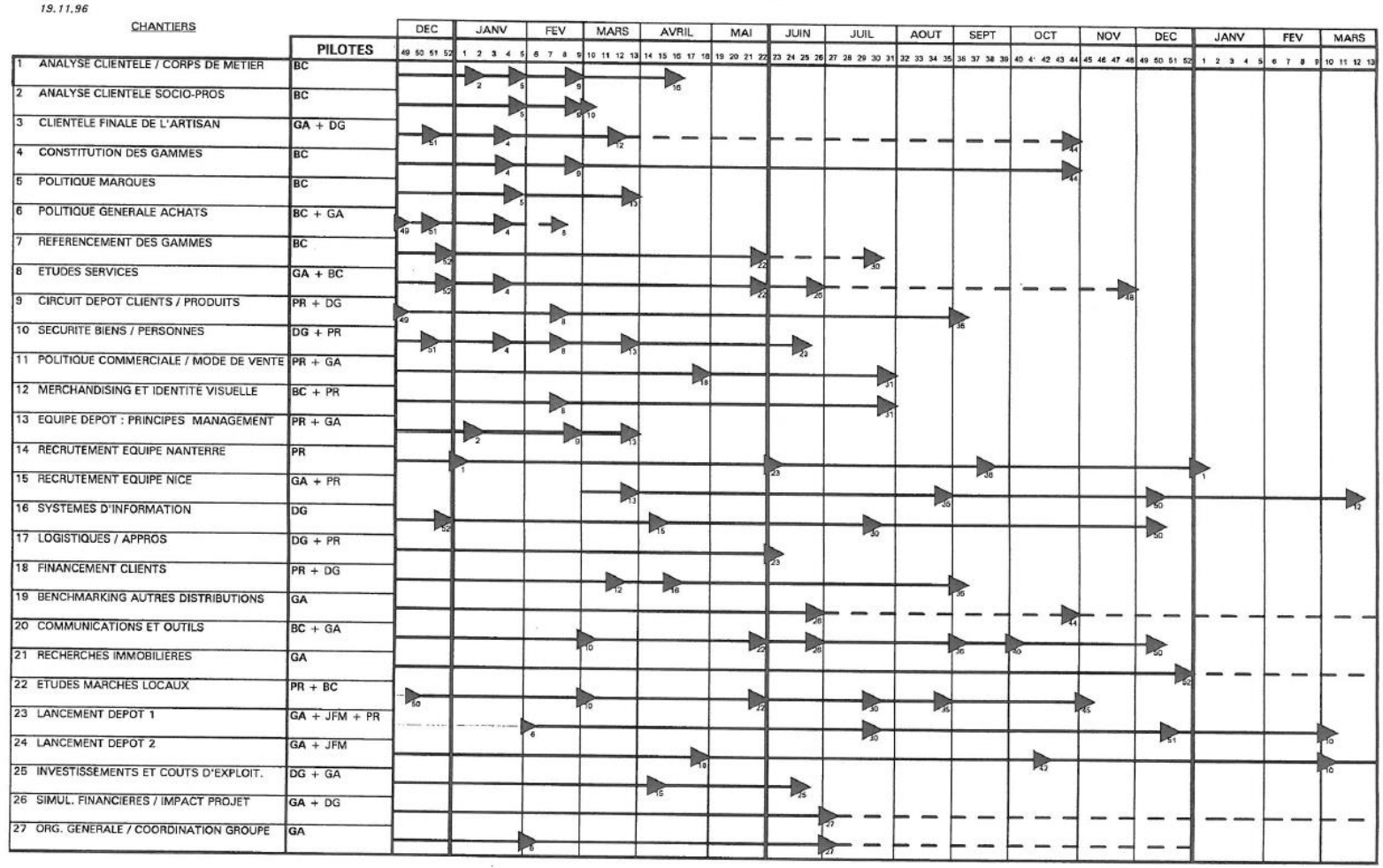

Figure 2. "Résumé de chacun des 27 chantiers », 19/11/1996 (source : archives Gérard Aspar)

Chaque élément du modèle a été testé rigoureusement et confronté à des modes de validation analytique. Par exemple, si la passage d'un projet Business to Consumer à Business to Business vient de la contrainte imposée par la loi Raffarin, la construction de l'offre B to B a bénéficié de l'expertise de personnes internes, notamment Maurice Manceau qui a pu auditer la modification du projet vers les professionnels. Cela a impliqué de revoir le choix de la gamme. Pour chaque métier, il faut alors 
méthodiquement identifier les principaux besoins des artisans à partir des analyses de marchés [CAR $18]$ et les référencer, négocier des prix avec les fournisseurs, etc.

\section{Conclusion}

Dans la présente contribution nous nous sommes attachés à rendre compte de la genèse de la Plateforme du bâtiment. Il apparaît que cette dernière résulte du déploiement conjoint de la rationalité créative et de la rationalité analytique, toutes deux intervenant, bien qu'à des étapes différentes, pour produire un concept puis le faire évoluer afin qu'il réponde à un système de contraintes hétérogènes. Ceci nous conduit à mettre en avant quatre points de conclusion.

En premier lieu, l'histoire de la genèse de la Plateforme du bâtiment permet de souligner qu'il ne suffit pas de faire émerger un concept inédit pour innover, on ne saurait occulter que la forme finale prise par l'innovation résulte de la mise à l'épreuve du concept imaginé. Cette mise à l'épreuve prend la forme de la confrontation du concept initial (c'est-à-dire tel qu'esquissé en 1996) aux contraintes du processus de conception. La prise en compte de ces contraintes donne lieu, comme on a pu le constater dans les lignes qui précèdent, à des réactions différenciées. Si la prise en compte de la Loi Raffarin donne lieu à un traitement que nous pourrions qualifier d'opportuniste (elle est «contournée » quasi instantanément en décidant de changer la cible) d'autres, au contraire, sont à l'origine d'un recueil de données permettant de mieux saisir qui sont, par exemple, les petits artisans, quels sont leurs besoins, leurs difficultés.

En second lieu, il apparait que la rationalité créative n'opère pas que dans la genèse d'innovation de produit ou procédé mais aussi pour une innovation marketing relative à un nouveau mode de distribution. La Plateforme du bâtiment est le fruit d'une synthèse ingénieuse, rendue possible par une curiosité et une ouverture qui permettent d'étendre le champ des possibles.

Troisièmement, l'exemple de la Plateforme souligne que l'innovation résulte de la mise en œuvre de deux formes de rationalité créative et analytique. Si la rationalité créative est emblématique de l'ouverture des possibles, la rationalité analytique permet quant-à elle d'éprouver les idées issues de la rationalité créative, de structurer les étapes du déploiement du concept, etc., Des formes de rationalités différentes pour des rôles distincts et nécessaires à l'innovation.

Enfin, quatrième et dernier point : la genèse de la Plateforme du bâtiment réactualise la question du lien entre innovation et société. En mettant l'accent sur le déploiement de la rationalité créative on confirme la thèse selon laquelle l'émergence d'un concept innovant n'arrive pas dans un désert et ne procède pas ex nihilo. De même la prise en compte des contraintes de l'environnement participe de la définition de la forme finale que prendra l'innovation. L'innovation possède donc une inscription sociale et temporelle, et les « règles du jeu » évoluant, il est possible d'affirmer que «chaque époque à les innovations de son temps $»^{11}$. Les innovations sont, pour le dire autrement, la matérialisation des conditions qui les ont vu naître, même si ces dernières s'effacent dans le temps.

\section{Bibliographie}

[ALT 00] ALTER N., L’innovation ordinaire, Presses Universitaires de France ? Paris, 2000.

[BEF 17] BEFFA J-L., Se transformer ou mourir, Editions du Seuil, Paris, 2017.

${ }^{11}$ De même que, par un jeu de co-évolution, chaque époque porte en elle les stigmates des innovations de son temps. 
[CHO 19] CHOUTEAU M., FOREST J., NGUYEN C., «Concevoir en donnant du sens à l'innovation : l'approche P.S.I », dans J.Didier, D.Leuba, Y.C Lequin (dir), Didactique de la conception, UTBM Editions, Belfort, à paraitre, 2019.

[CHR 13] CHRISTENSEN C., DYER J., GREGERSEN H., Le gène de l'innovateur : Cinq compétences qui font la différence, Pearson France, 2013.

[FAL 90] FALZON P., BISSERET A., BONNARDEL N., DARSES F., DETIENNE F., VISSER W., Les activités de conception : l'approche de l'ergonomie cognitive, Colloque Recherches sur le Design, Compiègne, 17-19 octobre 1990.

[FED 11] Fédération Française du Bâtiment., « Grands chiffres et figures », Constructif, n²9, 2011.

[FOR 18] FOREST J., «Vers des organisations ouvertes à l'imprévu », La Recherche, Chronique Innovation, mai 2018. $\mathrm{N}^{\circ} 535$, p. 36, 2018.

[FOR 17] FOREST J., Creative Rationality and Innovation, Smart Innovation set, volume 14, WILEY-ISTE Editions, London, 2017.

[FOR 01] FOREST J, MEHIER C., «J.R.COMMONS and H.A.SIMON on the concept of Rationality », Journal of Economic Issues, September 2001. vol XXXV, n³, p. 591-605, 2001.

[KOE 64] KOESTLER A., The act of creation, Arkana, London, 1964.

[LSA 99] LSA., «La Plateforme : tout le bâtiment en libre-service », LSA Commerce \& Consommation, 01/04/1999. En ligne : https://www.lsa-conso.fr/la-Plateforme-tout-le-bâtiment-en-libre-service,32386. Consulté le 17 septembre 2018, 1999.

[PER 15] PERRIN C., « Un million d'artisans. La situation actuelle de l'artisanat français dans une perspective historique », Marché et organisations, 2015/3 (n² 24), p. 15-36, 2015.

[SIM 57] SIMON H.A., Models of Man: Social and Rational. Mathematical Essays on Rational Behavior in a Social Setting, Wiley Editions, New York, 1957.

\section{Archives Saint Gobain et privées}

[S.N 08a] S.N., «La Plateforme du bâtiment à Argenteuil », Le Mois, 146, octobre 2008 : Archives Saint Gobain : DOC REV 00862/312.061.

[S.N. 08b] S.N., Vidéo des 10 ans VTS-01-1, 2008 : Archives privées.

[S.N 97] S.N., Note de synthèse du projet, 17 décembre 1997 : Archives Saint Gobain : SGBDF 00006/28.

[S.N 98] S.N., Tarifs 1998, La Plateforme du bâtiment, 1998.

\section{Liste des interviews}

[ASP 17] ASPAR G., directeur général \& fondateur de la Plateforme du Bâtiment distribution de 1996 à 1999. Entretien via skype par Ludovic Viévard, 20 novembre 2017.

[ASP 18] ASPAR G., directeur général \& fondateur de la Plateforme du Bâtiment distribution de 1996 à 1999. Entretien en présentiel par Ludovic Viévard, Paris, 15 juin 2018.

[RIC 18] RICHARD P., directeur général de la Plateforme du Bâtiment en France et des enseignes espagnoles de SaintGobain Building Distribution de 1997 à 2007. Entretien en présentiel par Ludovic Viévard, Paris, 20 février 2018.

[MAN 18] MANCEAU M., directeur délégué de Saint-Gobain Habitat depuis 2008. Entretien en présentiel par Ludovic Viévard, Paris, 21 février 2018.

[CAR 18] CARON B., responsable marketing et achats de la Plateforme du Bâtiment, 1997 à 1998. Entretien en présentiel par Ludovic Viévard, Suresnes, 14 mars 2018.

[BEF 18] BEFFA J-L., président directeur général du Groupe Saint-Gobain de 1986 à 2007. Entretien en présentiel par Ludovic Viévard, Paris, 6 avril 2018.

[VEN 18] VENZAL P., directeur des magasins Plateforme du Bâtiment de Nanterre (X-X) et Aubervilliers (X-X). Entretien en présentiel par Ludovic Viévard, le 21 juin 2018, à Paris. 